\title{
Lexikon der österreichischen Provenienzforschung
}

\section{https://www.lexikon-provenienzforschung.org/}

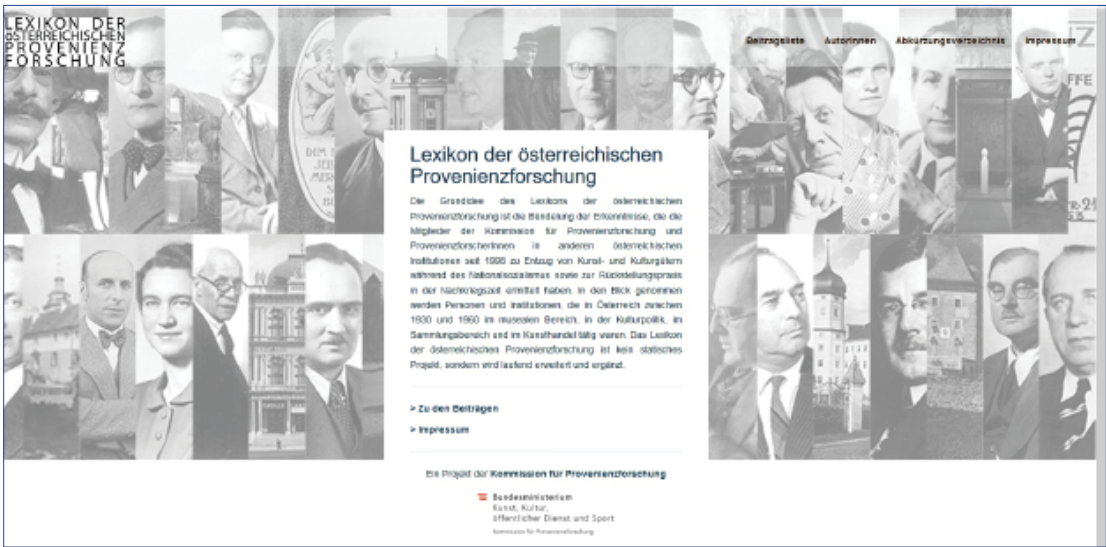

Zum Entzug von Kunst- und Kulturgütern während der Zeit des Nationalsozialismus sowie zur Rückstellungspraxis in der Nachkriegszeit wird in Österreich seit 1998 systematisch geforscht - im selben Jahr erfolgten die Gründung der Österreichischen Kommission für Provenienzforschung, die Verabschiedung der „Washington Conference Principles on Nazi-Confiscated Art“ (https://www.state.gov/washington-conference-principles-on-nazi-confiscated-art/) und die Beschlussfassung des „Bundesgesetz über die Rückgabe von Kunstgegenständen und sonstigen beweglichem Kulturgut aus den österreichischen Bundesmuseen und Sammlungen und aus dem sonstigen Bundeseigentum (Kunstrückgabegesetz - KRG)“ (https://www.ris.bka.gv.at/GeltendeFassung. $w x e$ ? Abfrage=Bundesnormen\&Gesetzesnummer=10010094).

In den vergangenen zwei Jahrzehnten wurde über den nationalsozialistischen Kunstraub in Österreich von Mitgliedern der Kommission für Provenienzforschung und auch von Provenienzforscherinnen und Provenienzforschern an anderen österreichischen Institutionen, wie z.B. den Universitätsbibliotheken der Medizinischen Universität Wien und der Universität Wien, eine Vielzahl an wissenschaftlichen Erkenntnissen gewonnen, die in zahlreichen Publikationen oder auf diversen Websites verstreut ihren Niederschlag gefunden haben; eine aktuelle bzw. laufend aktualisierte Zusammenschau, die einen fundierten Einblick in die gesamte Thematik bieten würde, fehlt allerdings bisher. Aus dieser unbefriedigenden Ausgangslage heraus entstand die Idee zu einem „Lexikon der österreichischen 
Provenienzforschung" (LÖPF), in dem die Forschungsergebnisse zu Personen und Institutionen, die zirka zwischen 1930 und 1960 in Museen, in der Kunst- und Kulturpolitik, im Bereich des Sammelns sowie des Handelns mit Kunst- und Kulturgütern in Österreich tätig waren, disziplin- und institutionsübergreifend zusammengeführt und in einheitlicher Form publiziert werden. Das LÖPF wurde anlässlich des 20jährigen Bestehens der „Washington Principles" und der Kommission für Provenienzforschung im Jänner 2019 an der Fachbereichsbibliothek Zeitgeschichte der Universität Wien präsentiert.

Die Herausgeberin des LÖPF ist die Kommission für Provenienzforschung beim Bundesministerium Kunst, Kultur, öffentlicher Dienst und Sport (https://www.provenienzforschung.gv.at). Idee, Konzeption und Projektentwicklung stammen von Pia Schölnberger und Leonhard Weidinger, die zwischen 2014 und 2017 auch für die wissenschaftliche Redaktion verantwortlich zeichneten; seit 2018 liegt diese bei Konstantin Ferihumer und Susanne Hehenberger.

Das LÖPF wurde von Anfang an als Online-Publikation konzipiert, was mehrere Vorteile gegenüber einer Print-Publikation mit sich bringt:

Das Online-Lexikon beinhaltet auch Informationen zu Personen und Institutionen, die in anderen Publikationen, insbesondere Zeitschriftenartikeln oder Beiträgen in Sammelbänden, aus Platzgründen nur erwähnt werden können, sowie Personen und Institutionen, die im Hintergrund der Raub- und Enteignungsprozesse mitgewirkt haben oder eingebunden waren (z.B. Abwicklerinnen und Abwickler, Juristinnen und Juristen).

Die Beiträge des LÖPF können laufend um neue erweitert bzw. bereits veröffentlichte Artikel um neue Erkenntnisse ergänzt werden.

Die Konzeption des LÖPF als Internetportal bietet auch die Möglichkeit, Zusammenhänge in personeller und institutioneller Hinsicht, die Opfer, Täterinnen und Täter oder mitwirkende Organisationen betreffen, durch Verlinkungen besser sichtbar zu machen, sowie die vielfältigen Verstrickungen aufzuzeigen.

Das LÖPF steht als wertvolle Informationsquelle online für alle Interessierten kostenfrei zur Verfügung, weil sämtliche Beiträge im Volltext Open Access veröffentlicht wurden und werden.

Und nicht zuletzt stellt das LÖPF über den Kreis der an den Ergebnissen der Provenienzforschung interessierten Personen hinausgehend als global zugängliches Portal für potentiell Erbberechtigte sowie für Nachfahrinnen und Nachfahren von Opfern des Kunstraubes eine wertvolle Quelle dar.

Das LÖPF verfügt über einen schlanken Webauftritt, der allerdings alle wichtigen Informationen zum Lexikon über die Navigation, die sich in vier 
Menüpunkte gliedert, sowie eine einfach zu handhabende Rechercheoberfläche bietet.

Der Menüpunkt „Beitragsliste“ bietet eine alphabetische Übersicht über die im LÖPF veröffentlichten Artikel, wobei man von jedem Begriff bzw. Personennamen mit einem Klick direkt zur dazugehörigen Information gelangt. Ein Desiderat stellt eine strukturierte (alphabetische) Einstiegsoption dar, die einen gezielten Zugang zu Begriffen mit einem bestimmten Anfangsbuchstaben ermöglicht.

Mit Stand 31. Jänner 2020 beinhaltet das LÖPF 265 Beiträge (siehe Kasten am Ende der Rezension), die einheitlich strukturiert sind und aus zwei Teilen - einem Lexikoneintrag sowie Quellen und Literatur - bestehen; auf einer Unterseite findet sich der Lexikonbeitrag, auf einer weiteren sind die jeweils maßgeblichen Quellen sowie Literaturstellen aufgelistet. Die Artikel sind mit Schlagwörtern versehen, über die man alle Beiträge eines bestimmten Schlagworts direkt aufrufen kann, sowie mit der Information, wer den entsprechenden Beitrag geschrieben hat, und wann dieser online gestellt wurde. So sind etwa unter dem Schlagwort „Bibliothek“ zehn, unter „Bibliothekarln" neun Beiträge verzeichnet. Bisher nicht vorhanden ist ein Verzeichnis aller verwendeten Schlagwörter, sodass derzeit leider keine gezielte Suchmöglichkeit nach Beiträgen zu einem bestimmten Thema besteht.

Von den bisher veröffentlichten 265 Artikeln entfallen zirka 200 in die Kategorie „Personeneinträge“; diese beinhalten biografische Informationen zu Opfern oder Tätern des nationalsozialistischen Kunstraubes. In den weiteren 65 Beiträgen werden Institutionen thematisiert, die ein breites Spektrum abdecken: von Institutionen und Firmen, die Opfer des nationalsozialistischen Kunstraubes waren (z.B. Akademischer Verein jüdischer Mediziner Wien, Antiquitätenhandlung J. Glückselig \& Sohn, Buch-, Kunst- und Musikalienhandel Moritz Perles, Brüder Suschitzky Anzengruber Verlag), über nationalsozialistische Einrichtungen, die für den Kunstraub verantwortlich waren (z.B. Arisierungskommission der Wiener Photographeninnung, Bergungsstelle Gaming, Vermögensverkehrsstelle, Verwaltungsstelle für jüdisches Umzugsgut der Gestapo - Vugesta, Zentraldepot für beschlagnahmte Sammlungen) bis zu jenen Institutionen, an die NS-Raubgut gelangt ist und die Provenienzforschung betreiben (z.B. Akademie der bildenden Künste Wien, Medizinische Universität Wien, Oberösterreichisches Landesmuseum, Österreichische Galerie, Technisches Museum, Tiroler Landesmuseum Ferdinandeum, Universitätsbibliothek Wien).

195 Beiträge, die am 7. Jänner 2019 freigeschaltet wurden, bilden den Nukleus des LÖPF; weitere 70 Beiträge wurden im Laufe des Jahres 2019 ergänzt. Mit den bisher publizierten Artikeln konnte das LÖPF einen be- 
merkenswerten Startpunkt setzen, auch wenn damit erst ein kleiner Teil der relevanten Themen zu Kunst- und Kulturraub in Österreich abgedeckt wurde, wie etwa beim Spezialthema des NS-Bücherraubes konstatiert werden konnte. So etwa wurden erst zu elf von mehr als 160 Personen bzw. Institutionen, die in einer im Dezember 2015 von Christina Köstner-Pemsel und Markus Stumpf erstellten Tabelle zu abgeschlossenen und offenen Restitutionsfälle von zehn wissenschaftlichen Bibliotheken in Österreich verzeichnet wurden (Erich Bien, Alois Fantl, Hugo Theodor Horwitz, Raoul Fernand Jellinek-Mercedes, Antiquariat Hans Peter Kraus, Ernst Moriz Kronfeld, Wilhelm Müller-Hofmann, Carl Julius Rothberger, Fritz Saxl, Antiquariat Philipp \& Adele Suschitzky, Maximilian Weinberger), Beiträge im LÖPF veröffentlicht (https://www.univie.ac.at/voeb/fileadmin/Dateien/ Kommissionen/AG_Provenienzforschung/201512_Liste_Restitutionsfaelle.pdf).

Es wird also von den Verantwortlichen des LÖPF in den kommenden Jahren unter Beweis zu stellen sein, ob das zukunftsweisende Konzept der laufenden Erweiterung und Aktualisierung auch nachhaltig in die Realität umgesetzt werden kann.

Um sich rascher über etwaige Nachträge und Verbesserungen informieren zu können, wäre es wünschenswert, dass neue Beiträge als eigener Menüpunkt auf der Startseite des LÖPF separat ausgewiesen werden und eventuell von interessierten Personen ein Benachrichtigungsdienst abonniert werden kann.

Unter einem weiteren Menüpunkt sind die am LÖPF beteiligen Autorinnen und Autoren ebenfalls in alphabetischer Reihenfolge, jeweils mit einer Kurzbiografie, verzeichnet. 31 Expertinnen und Experten, die durchwegs über eine große Erfahrung auf dem Gebiet der NS-Provenienzforschung und der Restitution verfügen, zeichnen bislang für die einzelnen Beiträge verantwortlich: Gabriele Anderl, Nadine Bauer, Sarah Bock, Sonia Buchroithner, Julia EßI, Konstantin Ferihumer, Lisa Frank, Katinka Gratzer-Baumgärtner, Christina Gschiel, Susanne Hehenberger, Olivia Kaiser, Birgit Kirchmayr, Christian Klösch, Christina Köstner-Pemsel, Karin Leitner-Ruhe, Andreas Liška-Birk, Sabine Loitfellner, Monika Löscher, Monika Mayer, Walter Mentzel, Gerhard Milchram, Sonja Niederacher, Lena Nothdurfter, Anneliese Schallmeiner, René Schober, Pia Schölnberger, Claudia Sporer-Heis, Claudia Spring, Anita Stelzl-Gallian, Markus Stumpf und Leonhard Weidinger.

Vermisst wurde vom Rezensenten die Möglichkeit, über einen direkten Link vom jeweiligen Namen sämtliche Beiträge einer Autorin oder eines Autors direkt aufrufen zu können. 
Das Abkürzungsverzeichnis des LÖPF ist über einen weiteren Menüpunkt aufrufbar und beinhaltet an die 100 Abkürzungen und deren Bedeutung für Fachtermini und Institutionen, die für viele Beiträge relevant sind.

Unter dem Menüpunkt „Impressum“ findet sich unter „Kontakt“ auch die Möglichkeit, unkompliziert eine Anfrage an die Redaktion des LÖPF zu richten, sowie ein Beispiel für die empfohlene Zitierweise für einzelne Beiträge des LÖPF.

Zusammenfassend ist festzuhalten, dass mit dem „Lexikon der österreichischen Provenienzforschung" ein wichtiges Desiderat für Forscherinnen und Forscher, die sich mit dem nationalsozialistischen Kunst- und Kulturgüterraub und der Restitution beschäftigen, behoben wurde. Deshalb ist es den Verantwortlichen des LÖPF zu wünschen, dass sich weitere Autorinnen und Autoren vor allem jener Einrichtungen, die bisher noch nicht im LÖPF vertreten sind, für die Mitarbeit finden und das Konzept der kontinuierlichen Erweiterung und Aktualisierung der Inhalte nachhaltig umgesetzt werden kann.

Mag. Bruno Bauer

ORCID: https://orcid.org/0000-0002-4729-331X Medizinische Universität Wien, Universitätsbibliothek

E-Mail: bruno.bauer@meduniwien.ac.at

\section{Lexikon der österreichischen Provenienzforschung (Liste der 265 Lemmata, Stand: 31.01.2020)}

A

Adelhart, Michaela Thekla

Adler, Marianne (Mary Ann)

Adriani, Gert

Akademie der bildenden Künste in Wien

Akademischer Verein jüdischer

Mediziner in Wien

Albertina

Almas Dietrich, Maria

Alsberg, Max

Altaussee, Salzbergwerk

Ambras, Schloss

Arisierungskommission der

Wiener Photographeninnung

Arnstein, Alfred

Augustinerkeller, Wien
B

Baczewski, Max Leopold

Baldass, Ludwig

Balke, Franz

Baranyi, Karl

Bauer, Samuel

Bayer, Alexander

Beer, Alexander

Berger, Josefine

Bergthold, Emmerich

Bibliothek der Gesellschaft der Ärzte

in Wien

Bibliothek Sassenbach

Bien, Erich Arthur

Blauhorn, Josef

Blum, Babette 
Bokh, Leo

Brunner, Maria

Buchwieser, Helene Elisabeth

Leopoldine

Buschbeck, Ernst

C

Chefez, Nachim (Nachum)

Christian, Viktor

D

Degenhart, Bernhard

Demel (von Elswehr), Hans

Detoni, Josef Leopold Anton

Drey, Julius

Duschnitz, Willibald

Dworak, Josef Zdenko

Dworak, Josef Zdenko junior,

Möbeltransport-Lagerhaus

Dworschak, Fritz

E

Ecker, Karl

Eder, Maximilian

Egger, Ernst

Ehrenfest-Egger, Regine

Eichler, Fritz (Friedrich)

Eigenberger, Robert

Eissler, Hermann

Engel, Rudolf

Epstein, Jehudo

Ernst, Richard

Exner, Anton

Exner, Walter

Eymer, August Friedrich

$\mathbf{F}$

Faltis, Otto

Fantl, Alois, Buchhandlung und modernes Antiquariat
Fargel, Julius

Feiler, Karl

Feldmann, Arthur

Felsenstein, Novella

Feuer, Adella

Fiala, Erich Franz

Fleischmann, Benno Carl Richard

Fleischner, Josef Isidor

Freund, Julius

Friedberg, Schloss

Friess, Eugenie

Frodl, Walter

Fügen, Schloss

G

Gaming

Garzarolli-Thurnlackh, Karl

Gasselseder, Elisabeth Maria

Gayrsperg, Ivo Hans

Gerstl, Siegfried

Getzinger, Alois

Geyer, Emil

Gilhofer und Ranschburg

Glückselig, Auktionshaus für

Altertümer

Glückselig, J. \& Sohn,

Antiquitätenhandlung

Glückselig, Rosa

Goldmann, David

Grabner, Ottilie

Gregor, Joseph

Grimschitz, Bruno

Grosz, Alexander

Grósz, August Joseph Benedict

Grünbaum, Elisabeth

Grünbaum, Franz Friedrich

Grünebaum, Moriz

Gussenbauer, Hildegard

Gutmann, Ludwig 
H

Hadamowsky, Franz

Haim, Emil \& Co, Akademische

Verlags- und Versandbuchhandlung

Hamburger-Löw, Marianne

Hamel, Oskar

Hareiter, Karl

Hassel, Margarethe

Heigl, Paul

Heim, Heinrich

Heissfeld, Lotte

Heissfeld, Valerie

Herzfeld, Paul David

Heumann, Carl

Historisches Museum der

österreichischen Eisenbahnen

Historisches Museum der Stadt Wien

Horwitz, Hugo Theodor

Hupka, Josef Franz

I

Immendorf, Schloss

Irtl, Adolf

Israelitisches Blindeninstitut Hohe Warte Iwnicki, Emil

\section{J}

Jancsy, Anton

Jellinek, Stefan

Jellinek-Mercedes, Raoul Fernand

Joanneum

Jonas, Robert

Jung, Hubert Wilhelm

K

Kantor, Eva Ida Benjamine

Kastner, Walther

Katzler, Johannes

Kauftheil, Hermann

Kellner, Maximilian
Kende, Albert

Kitschelt, Lothar Rudolf Moriz

Klapsia, Heinrich

Klein-Engersdorf, Pfarrhof

Klinkhoff, Johann

Klosterneuburg, Stift

Kolb, Arnold Herbert

Kolisch, Hermann Emanuel

Körbel, Hans

Kraus, Hans Peter

Kremsmünster, Reichskunstdepot

Krenn, Karl

Kris, Ernst

Kronfeld, Ernst Moriz

Kuderna, Josef

Kulka, Adele

Kulka, Richard

Kummerlöwe, Hans

Kunst und Wohnung, R. Lorenz

GesmbH

Kunsthistorisches Museum, Wien

Kunstmuseum Linz

Kurzel-Runtscheiner, Erich

Kutscher, Anna Chane

L

Lagler-Wustl, Richardis

Lányi, Richard

Lauffen, Salzbergwerk

Leinkauf, Hans

Lejeune, Fritz

Lhotsky, Alphons

Lichtenwerth, Burg

Lilienthal, Cäcilie

Lindemann, Moritz

Löbl, Otto

Löwi, Richard

Löwy, Franz

Luithlen, Victor

Lukacs, Mathilde 
M

Maier, Rudolf

Mandl-Maldenau, Max

Marmorek, Hugo

Matzen, Schloss

Mautner, Anna Constanze

Mayer, Karl

Meisels, Chaim Salomon

Meyerweissflog, Fritz Georg

Müller-Hofmann, Wilhelm

$\mathbf{N}$

Nadler, Richard

Nagler, Ferdinand

Naturhistorisches Museum Wien

Nehammer, Karoline

Neuburger, Max

Neumann, L. T.

Neurath, Ludwig

Nohynek, Gustav

Noll, Rudolf

Novotny, Fritz

O

Oberhammer, Vinzenz

Oberösterreichisches Landesmuseum

Ortner, Karl

Österreichische Galerie

Österreichische Mediathek

Österreichisches Museum für

angewandte Kunst

$\mathbf{P}$

Pächter, Adele

Penizek, Melanie

Perles, Moritz, Buch-, Kunst- und

Musikalienhandel

Pfannl, Mathilde

Poch-Kalous, Margarethe

Pollack, Ernst
Pollak, Wilhelm

Posse, Hans

Post- und Telegraphen Museum Wien

Primavesi, Eugen

Pulkau, Pfarrhof

Purgstall an der Erlauf, Schloss

$\mathbf{R}$

Reich, Otto Franz Mauriz

Reichel, Dr., Galerie

Reichel, Oskar

Reichenfeld, Moriz

Reichmann, Alois, Buch- und

Antiquariatshandlung

Reichmann, Armin

Reininghaus, Carl

Riedel, Erhard

Rochlitzer, Ludwig

Rosner, Emil

Rothberger, Heinrich

\section{S}

Saiko, Emanuel Georg Josef

Saxl, Felix Friedrich

Schalita, Arnold

Schatzker, Otto

Schein, Abraham Meier (Myron)

Schmidl, Marianne

Schneeberg, Schloss

Schönwörth, Schloss

Schütz, Hermine

Schützenhofer, Viktor

Schwaz, St. Martin

Seiberl, Herbert

Sennhein, René Richard

Seutter (von) Loetzen, Liselotte

Sigmundsried, Schloss

Siller, Josef

Sochor, Franz

Sonnberg, Schloss 
Sonnenschein, Ernst

Spany, Ferdinand

Spany, Ludmilla

Stams, Stift

Stein, Anna

Steinbach bei Göstling

Sternberg, Theodor

Stixenstein, Burg

Suschitzky, Brüder - Anzengruber Verlag (Wien-Leipzig), Buchhandlung-

Antiquariat-Leihbibliothek

\section{T}

Technisches Museum Wien

Thalheim, Schloss

Thorsch, Alfons

Thürnthal, Schloss

Tiroler Landesmuseum

Ferdinandeum

Tratzberg, Schloss

Tripp, Gertrude

U

Universitätsbibliothek der

Medizinischen Universität Wien

Universitätsbibliothek Wien

V

Vermögensverkehrsstelle

Voss, Hermann

VUGESTA
W

Wagner, Karl

Waldum, Johann

Weinberger, Maximilian

Weißenhofer, Anselm

Weitzmann, Oskar

Weitzmann, Salomon, Fotoatelier

Wellek, Walter

Wolf, Alfred. Reise- und

Versandbuchhandlung, Antiquariat

und Export

Wolf, Theodor

Wölfl, Maria

Wolfrum, Kunstverlag

Wunderlich, Isak

Wurzbach-Tannenberg, Alfred Wolfgang

$\mathbf{Z}$

Zelenka, Leopoldine

Zelenka, Leopoldine, Kunstantiquariat

Zels, Marianne

Zentraldepot für beschlagnahmte

Sammlungen

Zix, Ernst

Zweigbibliothek für Geschichte der

Medizin an der Universitätsbibliothek

der Medizinischen Universität Wien

DOI: https://doi.org/10.31263/voebm.v73i1.3431

(C) Bruno Bauer

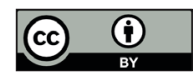

Dieses Werk ist - exkl. einzelner Logos und Abbildungen - lizenziert unter einer Creative-Commons-Lizenz Namensnennung 4.0 International 